\title{
Epilepsia refleja por agua caliente: a propósito de un caso en un lactante
}

Hot-water epilepsy: case report in an infant

\author{
Lic. Arantxa Vidal Esteban ${ }^{a}$, Lic. Pilar Galán del Río ${ }^{a}$ Dra. Teresa de Santos Moreno \\ Lic. Bárbara Muñoz Amatc y Dra. María J. Rivero Martín ${ }^{a}$
}

\section{RESUMEN}

Laepilepsia refleja por agua caliente es un tipo de convulsión poco frecuente cuya fisiopatología se desconoce. Estas crisis reflejas suelen iniciarse pocos segundos tras el contacto con el agua y, comúnmente, a temperaturas de $37-48^{\circ} \mathrm{C}$. Los automatismos y las crisis parciales complejas, con o singeneralización secundaria, son el modo de manifestación principal.

La exploración neurológica y el electroencefalograma intercrítico no suelen presentar alteraciones, lo que puede condicionar un retraso en el diagnóstico, por lo que es fundamental la sospecha clínica.

El tratamiento antiepiléptico se inicia cuando se asocia a otro tipo de epilepsia o cuando ciertas medidas sencillas, como el descenso de la temperatura del agua en el baño, no controlan las crisis. Es posible la desaparición espontánea. Cuando es necesaria la terapéutica farmacológica, existe, normalmente, buena respuesta.

Se presenta el caso de un lactante con diagnóstico de epilepsia refleja por agua caliente.

Palabras clave: convulsiones, epilepsia refleja, epilepsia refleja inducida por agua caliente.

\begin{abstract}
Hot-water epilepsy is a rare type of seizure whose pathophysiology is unknown. These reflex seizures usually begin a few seconds after contact with water, commonly at temperatures between $37-48{ }^{\circ} \mathrm{C}$. Automations and complex partial crises, with or without secondary generalization, are the main manifestation mode of this type of reflex epilepsies. Neurological examination and intercritical electroencephalography are usually normal, which may condition a delay in diagnosis, and the clinical suspicion is fundamental.

Antiepileptic treatment is initiated when associated with another type of epilepsy or when certain simple measures, such as lowering the water temperature in the bath, do not control crises. Spontaneous disappearance is possible; when pharmacological
\end{abstract}

a. Hospital Universitario de Fuenlabrada, Fuenlabrada, Madrid, España.

b. Hospital Universitario Clínico San Carlos, Madrid, España.

c. Hospital Universitario 12 de Octubre, Madrid, España.

Correspondencia:

Lic.Arantxa VidalEsteban:arantxa_vidal_esteban@hotmail.com

Financiamiento: Ninguno.

Conflicto de intereses: Ninguno que declarar.

Recibido: 2-3-2019

Aceptado: 3-9-2019 therapy is necessary, there is usually a good response. We present the case of an infant diagnosed with hot-water epilepsy.

Key words: seizures, reflex epilepsies, hot water-induced reflex epilepsy.

http: / / dx.doi.org/10.5546/ aap.2020.e183

Cómo citar: Vidal Esteban A, Galán del Río P, de Santos Moreno T, Muñoz Amat B, Rivero Martín MJ. Arch Argent Pediatr 2020; 118(2):e183-e187.

\section{INTRODUCCIÓN}

La epilepsia refleja por agua caliente es un síndrome epiléptico raro. Las crisis epilépticas aparecen, generalmente, a temperaturas que oscilan entre $37^{\circ} \mathrm{C}$ y $48^{\circ} \mathrm{C}$, y a los pocos segundos de tocar el agua (entre 30 segundos y 3 minutos). ${ }^{1,2}$ Las formas de presentación más frecuentes son los automatismos y las crisis parciales complejas, con o sin generalización secundaria. Este tipo de epilepsia ocurre, habitualmente, durante la primera década de la vida y es más frecuente en el sexo masculino $(3: 1){ }^{3}$

Es fundamental conocer este tipo de epilepsia en pediatría, ya que, aunque es infrecuente, su importancia radica en el diagnóstico sencillo al desencadenarse con la exposición de un estímulo táctil y térmico, así como la posible desaparición espontánea mediante medidas simples, sin necesidad, en todos los casos, de tratamiento farmacológico.

Se presenta el caso de un lactante con diagnóstico de epilepsia refleja por inmersión en agua caliente.

\section{CASO CLÍNICO}

Lactante de 7 meses sin antecedentes personales ni familiares de interés. El embarazo fue controlado con analíticas y ecografías prenatales, que no detectaron alteraciones significativas. Nació por parto eutócico y no presentó antecedentes perinatales reseñables. Tuvo un desarrollo psicomotor adecuado y calendario vacunal completo para su edad. 
Fue traído a Urgencias por presentar un episodio de hipotonía generalizada de 5 minutos de duración, que coincidió con el cambio del pañal, tras el baño, con recuperación completa y espontánea después de un corto período de somnolencia. No refirieron desconexión del medio, ni movimientos anómalos, ni mirada fija, ni cambios de coloración (sin cianosis ni palidez). No presentó cuadro febril ni clínica infecciosa asociada (ni en el momento actual ni en los días previos). Los padres refirieron que, en las últimas 3 semanas, había sufrido, de forma diaria, episodios similares que coincidían con el baño. El paciente presentaba una exploración física y neurológica sin alteraciones. Se solicitó analítica sanguínea, con resultado normal, excepto una anemia ferropénica no conocida previamente, y se solicitaron tóxicos en la orina, que fueron negativos. Además, se realizó un electrocardiograma, que resultó normal, y un ecocardiograma, en el que se observó únicamente un foramen oval permeable sin repercusión hemodinámica. Ante la clínica descrita, se decidió el ingreso del paciente en la planta de hospitalización para la vigilancia neurológica y para continuar con el estudio.
Se realizó un electroencefalograma en vigilia y con provocación del estímulo, que precipitó los episodios con resultado normal (actividad de fondo en vigilia de características normales para la edad del paciente y sin actividad epileptiforme con inmersión del paciente en el agua a $37^{\circ} \mathrm{C}$ y con aplicación del agua en el tronco) (Figura 1), y un estudio de imagen mediante ecografía transfontanelar, que no mostró alteraciones. Durante su ingreso en planta, presentó tres episodios similares, consistentes en hipotonía generalizada, con desviación de la mirada, cianosis perioral y desaturación asociada de hasta el $70 \%$, de 3-5 minutos de duración, que coincidió con la inmersión en agua caliente durante el baño (a los 30-60 segundos de la inmersión). Presentó somnolencia posterior de unos minutos de duración.

Ante la sospecha de epilepsia refleja por inmersión en agua caliente, se contactó con el hospital de referencia para la realización de registro de videoelectroencefalograma con provocación. En dicho estudio, se consiguió evidenciar el correlato electroclínico: se registraron dos crisis reflejas al baño con agua caliente, con semiología y cambios en el

FIGURA 1. Electroencefalograma intercrítico normal para la edad, con actividad basal bien diferenciada y simétrica tanto en la vigilia como en el sueño y sin evidencia de actividad epileptiforme intercrítica

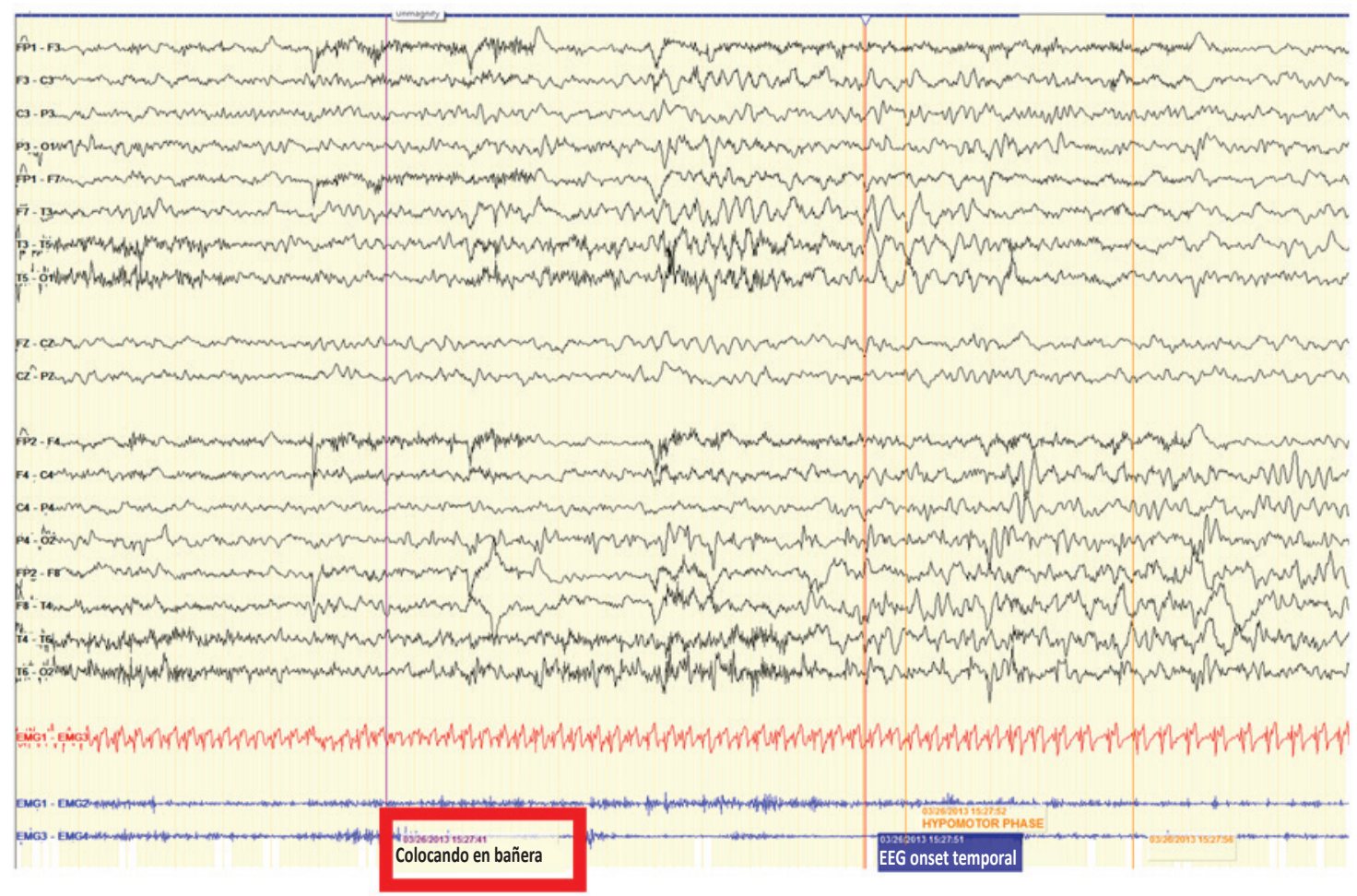


electroencefalograma estereotipados de origen temporal derecho, que se clasificaron como crisis hipomotoras y autonómicas, con registro intercrítico normal (Figura 2). Se realizó, además, una resonancia magnética, sin alteraciones.

Ante los hallazgos, se decidió iniciar el tratamiento con oxcarbazepina. Inicialmente, se indicó a los padres un seguimiento con controles mensuales y, luego, por buena respuesta al tratamiento farmacológico, sin aparición de nuevas crisis, controles trimestrales y semestrales.

\section{DISCUSIÓN}

Las crisis epilépticas reflejas se desencadenan por un estímulo sensorial precipitante específico. ${ }^{1}$ Pueden ser provocadas por factores de muy diversa índole (tales como hormonales, emocionales, sensoriales, eléctricos...). Por tanto, se diferencian de la epilepsia porque esta es una enfermedad cerebral definida por, al menos, dos crisis epilépticas no provocadas (o reflejas). La prevalencia de crisis reflejas en poblaciones de pacientes epilépticos varía, según las series, en torno al 1-6\%. ${ }^{4}$

La epilepsia refleja por agua caliente es un tipo de estas epilepsias reflejas. En 1945, Allen, en Nueva Zelanda, describió por primera vez la epilepsia refleja por agua caliente en un paciente de 10 años. Se han publicado casi 700 casos a nivel mundial, y las mayores series de casos (casi el $90 \%$ ) fueron reportadas en India (que suponía el 2,5-9 \% del total de las epilepsias) y Turquía. ${ }^{1,4}$ En la población occidental, se han descrito solo casos aislados. Las tasas elevadas en estos dos países, estaría influida, además de por la existencia de factores genéticos, por el ritual del baño. Parece existir evidencia de una base genética: se han identificado dos loci genéticos, uno en el cromosoma 4q24-q29 en una familia con 4 generaciones afectadas de forma autosómica dominante ${ }^{5} \mathrm{y}$ otro en el cromosoma 10q21.3-q22.3. ${ }^{4}$ En India y Turquía, existe un ritual de baño (echar agua caliente a temperatura que oscila entre $40^{\circ} \mathrm{C}$ y $50^{\circ} \mathrm{C}$ sobre la cabeza) con la creencia de que este tiene mayor beneficio cuanto mayor sea la temperatura del agua y cuanto más rápido se vierta. ${ }^{1,4}$ Esto podría explicar la base ambiental y sensorial por la que estos países tienen una mayor incidencia de este tipo de epilepsia.

En la epilepsia refleja por agua caliente, el tipo de crisis convulsiva más frecuente es la convulsión parcial compleja con o sin generalización secundaria o los automatismos

FIGURA 2. Crisis refleja al baño con agua caliente, con semiología y cambios en el electroencefalograma estereotipados de origen temporal derecho, que se clasifican como crisis hipomotoras y autonómicas, con registro intercrítico normal

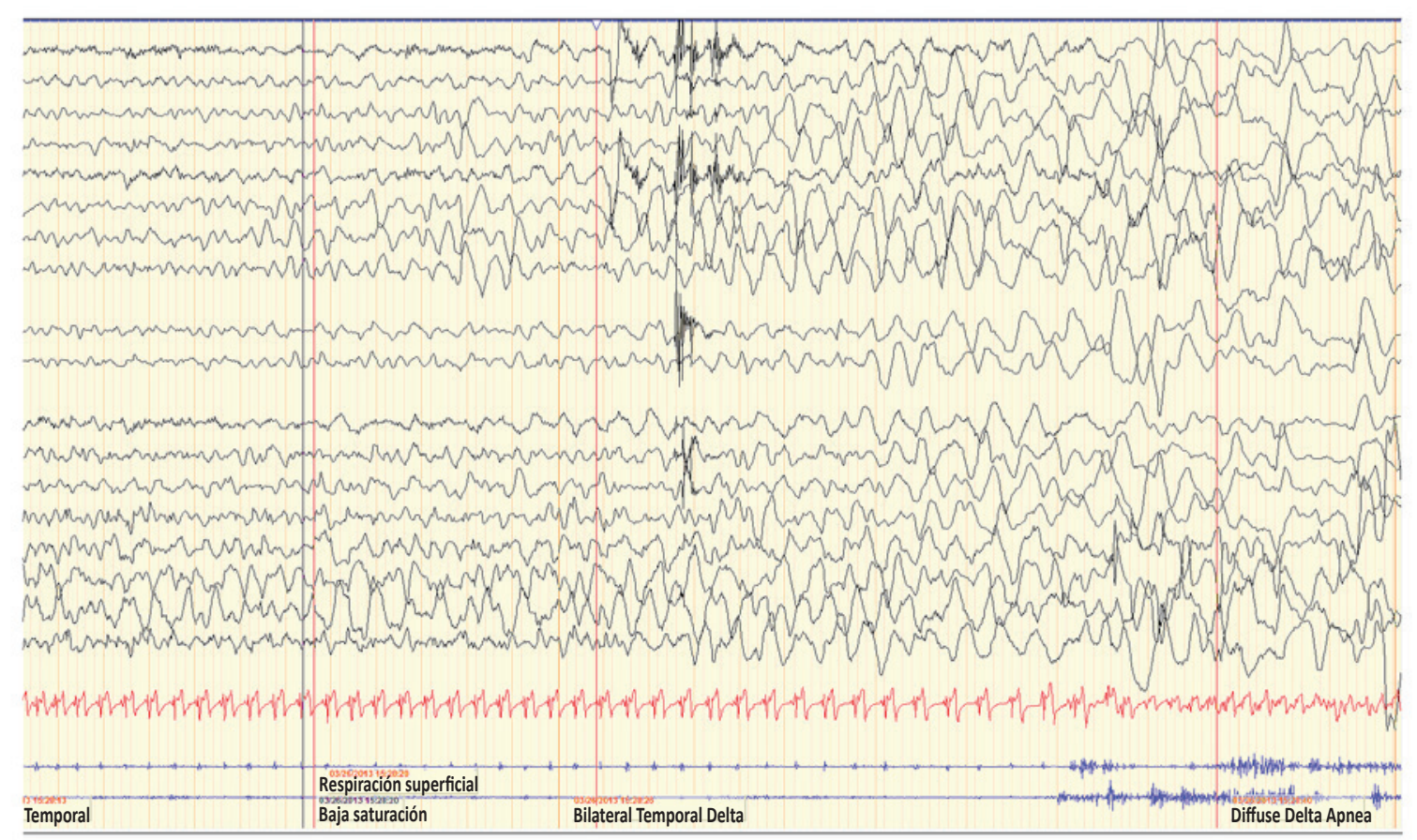


(el 67-71\%). En un $33 \%$, se presentan con crisis generalizadas y, predominantemente, tónicoclónicas. ${ }^{4}$ Un alto porcentaje de pacientes (el 16$62 \%)^{4}$ pueden asociar, además, crisis espontáneas no reflejas.

Aunque su fisiopatología es desconocida, sí que parece que existe un estímulo sensorial desencadenante que asocia, de forma simultánea, un estímulo táctil y térmico. ${ }^{1,2}$ Parece que el estímulo táctil es más importante que el cambio térmico que se produce en las estructuras internas, ya que las crisis aparecen a los pocos segundos (generalmente, entre 30 y 120 segundos) de contacto con el agua. Además, hay que destacar que las crisis solo se inducen a una temperatura específica, que oscila entre los $37^{\circ} \mathrm{C}$ y $\operatorname{los} 48^{\circ} \mathrm{C}$, lo que podría deberse a la respuesta fisiológica de descarga máxima de los termorreceptores existentes en el tejido cutáneo con temperaturas entre $32{ }^{\circ} \mathrm{C}$ y $45{ }^{\circ} \mathrm{C}$ (que no ocurre en temperaturas por encima de $45^{\circ} \mathrm{C}$ o inferiores a $\left.20-25{ }^{\circ} \mathrm{C}\right) .{ }^{3} \mathrm{El}$ hecho de que las crisis se inducen, en mayor medida, cuando se vierte el agua sobre la cabeza (y menos frecuentemente en otras zonas corporales) podría explicarse por la mayor sensibilidad de los receptores de las áreas corticales localizados en la región occipital, por la implicación de determinadas estructuras hipotalámicas que intervienen en la regulación de la temperatura y de centros autónomos situados en la profundidad de los lóbulos temporales ${ }^{2}$ (Figura 3).
En ocasiones, se plantea el diagnóstico diferencial con convulsiones parciales complejas con o sin generalización secundaria no reflejas y con el síncope vasovagal secundario a la temperatura elevada del agua. Sin embargo, durante estas crisis epilépticas reflejas, no se producen cambios en la frecuencia cardíaca, en la presión sanguínea ni en el electrocardiograma. ${ }^{6,7}$

El examen neurológico y el electroencefalograma intercrítico, frecuentemente, son normales, excepto en aquellos pacientes que asocian otro tipo de crisis epilépticas. En la mayoría de los casos descritos, con el electroencefalograma realizado durante la crisis, aparecen descritas, con más frecuencia, alteraciones con predominio en el hemisferio izquierdo y los lóbulos temporales.

Los estudios de neuroimagen son habitualmente normales. ${ }^{4}$ Pero hay que reseñar que, en la literatura, se han descrito casos de epilepsia refleja por agua caliente con lesiones estructurales en el hipocampo, displasia cortical o lesiones quísticas.

El tratamiento, inicialmente, se basa en medidas sencillas, como la disminución de la temperatura del agua o la modificación del método del baño, que pueden ser suficientes para controlar las crisis. Si estas medidas no son efectivas o si se presenta otro tipo de epilepsia concomitante, es necesario el inicio del tratamiento antiepiléptico. Es posible la desaparición espontánea, pero, si es necesario

FIGURA 3. Esquema general del sustrato fisiopatológico de la epilepsia refleja (fuente propia)

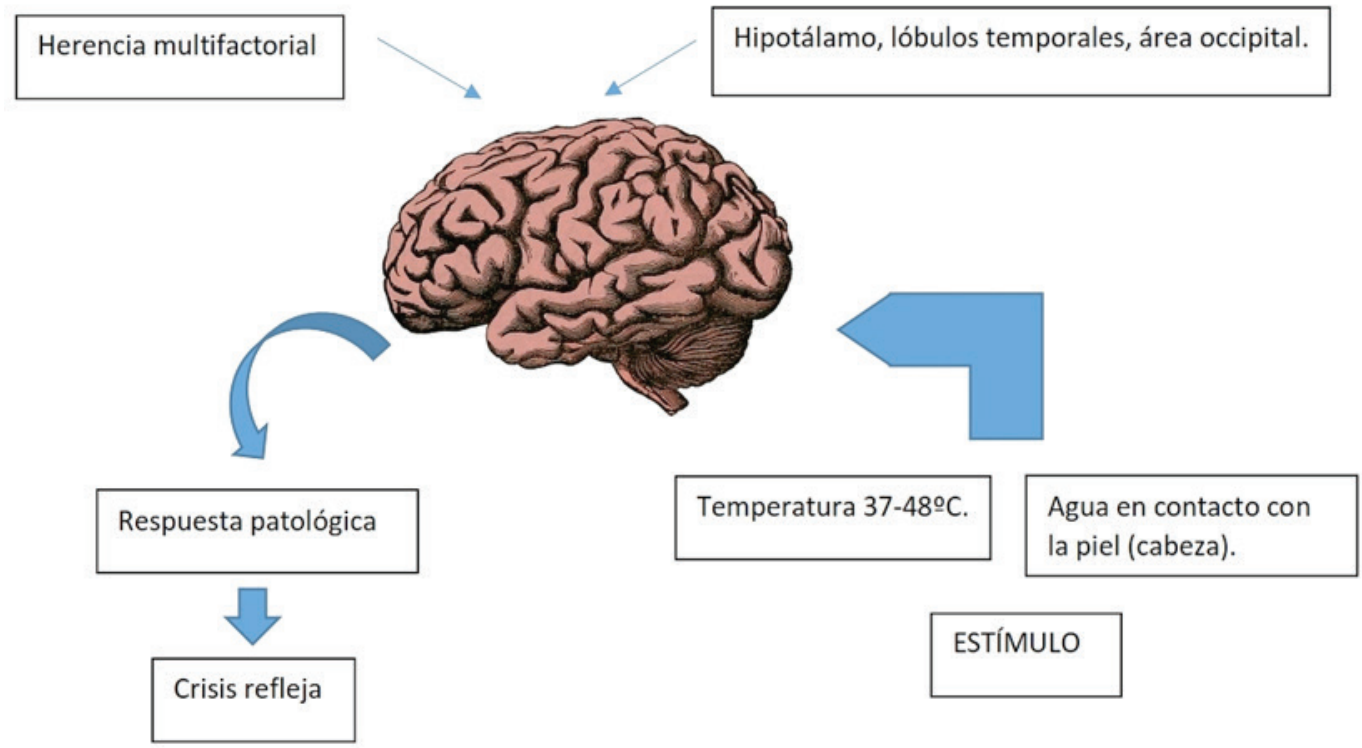


el tratamiento farmacológico, la respuesta es efectiva.

La epilepsia refleja por agua caliente presenta una respuesta clínica y electroencefalográfica característica cuando se expone al estímulo, lo que resulta importante para su identificación. Por tanto, debe ser conocida por los pediatras para realizar un diagnóstico temprano e iniciar un cambio en las medidas de baño y/o tratamiento farmacológico antiepiléptico.

\section{REFERENCIAS}

1. Pejaver R, Srinivasa V, Basavanthappa SP, Murthy CL. Hot water epilepsy: A form of reflex epilepsy - from infancy to adolescence. J Neurosci Rural Pract. 2015; 6(4):607-9.

2. Redzek Mudrinic T, Kavecan I, Starcevic IP, Stojadinovic A.
A case of infant hot water epilepsy: A clinical commentary with video sequences. Seizure. 2015; 31:5-6.

3. Satishchandra P, Dilipkumar S, Subbakrishna DK, Sinha S. Intermittent clobazam prophylaxis in hot water epilepsy is safe and effective: a prospective study. Epilepsy Res. 2014; 108(7):1238-42.

4. Vignoli A, Savini MN, La Briola F, Chiesa V, et al. Hot water epilepsy: a video case of European boy with positive family history and subsequent non-reflex epilepsy. Epileptic Disord. 2014; 16(1):96-100.

5. Patel M, Satishchandra P, Aravinda H, Bharath RD, et al. Hot water epilepsy: Phenotype and single photon emission computed tomography observations. Ann Indian Acad Neurol. 2014; 17(4):470-2.

6. Nunes Marta R, Cardoso Garcia P, Silva Fernandes V, Azevedo RochaS, et al. Epilepsia por agua caliente: una forma rara de epilepsia refleja. An Pediatr (Barc). 2011; 74(2):138-9.

7. Zeki G, Ilker IH, Hidir UU, Zeki O. Hot water epilepsy: seizure type, water temperature, EEG findings and treatment. Neurologist. 2010; 16(2):109-12. 